\title{
Coronary Artery Disease in Patients with Chronic Kidney Disease - A Review
}

\author{
M Faruque $^{1}$, AEM M Islam ${ }^{1}$, MA Rashid ${ }^{2}$, MS Haque ${ }^{1}$, F Rahman ${ }^{3}$, MG Kibria ${ }^{1}$, HILR Khan $^{1}$ \\ ${ }^{1}$ Department of Cardiology, Dhaka Medical College, Dhaka, ${ }^{2}$ Department of Anatomy, Bangladesh \\ Medical College, Dhaka. ${ }^{3}$ Department of Cardiology, Bangabandhu Sheikh Mujib \\ Medical University, Dhaka
}

(CVJ 2008; 1(1) : 97-104)

\begin{abstract}
Introduction
Cardiovascular diseases (CVD) are the leading causes of death in end-stage renal disease (ESRD) populations. There is increasing recognition that chronic kidney disease (CKD) of any degree portends a worsened prognosis for CAD patients and that long-term outlook in CKD patients is closely related to cardiovascular events. The cardiovascular mortality rate is 20 to 40 times higher for adults on dialysis than for the general population. In treating patients on dialysis, nephrologists contend with the cumulative effects of the processes that cause renal failure, the generic consequences of globally disordered renal function, and the potential adverse effects of treatment or lack of treatment. The value of most therapeutic interventions is less certain for CKD versus nonCKD patients because the former have typically been excluded from randomized trials. This review discusses the epidemiology, pathogenesis, clinical manifestations, diagnostic work-up, treatment, prognosis, and multiple conundrums regarding $\mathrm{CAD}$ patients with CKD.
\end{abstract}

\section{Disease Burden}

The prevalence of $\mathrm{CAD}$ in $\mathrm{CKD}$ patients is high and is a major cause of morbidity and mortality.Chronic kidney disease has reached epidemic proportions. More than 320,000 patients of CKD required dialysis in ' 98 ; by 2010 , this number may exceed 650,000 patients ${ }^{1}$. Patients with mild to severe decrease in glomerular filtration rate (GFR) constitute a larger group, estimated in 1998 to be 13.3 million. Finally, there are many CKD patients without decreased GFR (5.9 million in 1998). ${ }^{1}$ In hemodialysis (HD) or peritoneal dialysis patients, prevalence is estimated at $40 \%$ with a $9 \%$ annual cardiovascular mortality. ${ }^{2}$
Renal transplant recipients (RTRs) have a lower CAD prevalence (15\%) with an annual cardiovascular mortality of $0.54 \%$, twice the general population. ${ }^{2}$ This lower prevalence may be due to patients with fewer comorbidities and lower CAD likelihood being chosen for transplantation. The need for nomenclature uniformity has led to a recent CKD classification based on estimated GFR.

\section{Cardiovascular events in CKD}

Chronic kidney disease increases cardiovascular event risk and portends a worse outcome if an event occurs. A study of 3,106 acute myocardial infarction (AMI) patients showed in-hospital mortality of $2 \%$ in normal renal function, $6 \%$ mild CKD, $14 \%$ moderate CKD, $21 \%$ severe CKD, and $30 \%$ in dialysis patients $(p<0.001)$ with a similar trend long-term. ${ }^{3}$

Patients with mild to moderate CKD and non-STsegment elevation acute coronary syndrome (ACS) showed higher 30- and 180-day mortality than nonCKD patients. ${ }^{4}$

One-year mortality after AMI was 59\% in dialysis patients and $24 \%$ in RTRs. In a post-AMI Medicare cohort comprised of 130,099 patients, one-year mortality was $24 \%$ without CKD, $46 \%$ with mild CKD, and $66 \%$ with moderate CKD. ${ }^{5}$

\section{Metabolic Derangements in CKD}

The internal milieu in CKD favors $\mathrm{CAD}$ development and raises the question whether renal insufficiency itself is a CAD risk factor.

Diabetes mellitus: Diabetic nephropathy accounts for $40 \%$ of new ESRD. The 2001 U.S. Renal Data System reported a progressive rise in diabetic ESRD from 500/million in 1991 to 700/million in $1999^{6}$. Moreover, chronic renal failure, independent of diabetes mellitus, is also associated with insulin 
resistance and glucose intolerance. Both are associated with the accumulation of advanced glycation end products that may induce cellular activation and endothelial damage, ultimately contributing to or even accelerating atherogenesis .

The mechanisms of vascular injury being beyond the scope of this review. There is considerable evidence that aggressive glucose control can significantly reduce microvascular and macrovascular complications of diabetes. Therefore, it is important not only to manage diabetes aggressively before the onset of renal disease but also to continue rigorous glycemic control after ESRD has developed. Because hypoglycemic reactions are more common in renal failure, there is a tendency for nephrologists and others who care for diabetics with ESRD to allow blood glucose concentrations to remain at levels that would be unacceptable in the absence of renal failure, thus sustaining the risk for vascular damage and atherogenesis. Sustained rigorous glycemic control before the onset, as well as after the initiation, of renal replacement therapies may be important in reducing cardiovascular morbidity and mortality in diabetic patients as has been shown by Wu et al. ${ }^{7}$ However, whether such benefits would be offset by excessive hypoglycemic complications needs to be investigated further.

Hypertension: Hypertension is a well-established risk factor not only for myocardial ischemia, atherogenesis, and coronary artery calcification but also for the development of left ventricular hypertrophy (LVH). By contributing to LVH, hypertension represents an important risk for sudden cardiac death. Hypertension prevalence in CKD ranges from $60 \%$ to $100 \%$, depending on CKD cause and severity and population studied.

Hypertension may cause or be an effect of CKD and confers an increased risk of cardiovascular events in all patient subsets. Forces on arterial and aortic walls produced by increased BP alter vascular endothelial cell function by inciting arterial oxidative stress and inflammatory responses. These effects in turn initiate the atherosclerotic process and stimulate various growth promoting peptides. The attendant endothelial dysfunction can lead to hypertrophy, hyperplasia, lipid incorporation, and calcification of the vascular smooth muscle and to associated reductions in vascular compliance and thus to tissue ischemia. The reduced elasticity of the aorta and peripheral arteries further contributes to increased cardiac work, LVH, and increased cardiovascular mortality in ESRD patients .

Hyperlipidemia: Hyperlipidemia prevalence is increased in CKD. Lipid abnormalities include increased serum triglycerides, very low-density lipoprotein, and intermediate density lipoprotein and decreased high-density lipoprotein. Elevated total cholesterol is found in 30\% of CKD patients without nephrotic syndrome and in $90 \%$ with nephritic syndrome compared with $20 \%$ of the general population. Low-density lipoprotein cholesterol, calculated by the Friedewald formula, assumes a 5 : 1 triglyceride/cholesterol ratio in the very lowdensity lipoprotein particle and ignores intermediate-density lipoprotein. Hemodialysis patients with normal calculated low-density lipoprotein cholesterol may actually have elevated intermediate-density lipoprotein cholesterol measured by ultracentrifugation. Because intermediate-density lipoprotein is atherogenic, the Friedewald formula probably underestimates the amount of atherogenic cholesterol and hence the risk of developing atherosclerosis. Increases in small and dense low-density lipoprotein and oxidized lowdensity lipoprotein cholesterol have been reported in dialysis patients, which may increase cardiovascular risk. Low serum cholesterol and albumin levels in HD patients probably reflect poor nutritional status and are independent predictors of increased mortality.

Calcium-phosphate product: Hemodialysis patients with hyperphosphatemia for 1 year and an elevated calcium phosphorous product have a higher mortality. Ganesh et al. ${ }^{8}$ found that hyperphosphatemic patients had $41 \%$ increased risk of cardiovascular death and $20 \%$ increased risk of sudden death. Hyperphosphatemia and high calcium phosphate product have been implicated in the pathogenesis of cardiovascular calcification, which may correlate with atherosclerotic plaque burden and increased AMI incidence.

Hyperhomocysteinemia: Hyperhomocysteinemia is associated with an increased incidence of cardiovascular events in ESRD and RTRs. ${ }^{9}$ Hyperhomocysteinemia may produce endothelial dysfunction; smooth muscle proliferation; platelet aggregation; activation of factors V, X, and XII; and 
modulation of tissue plasminogen activator, all creating a prothrombotic environment. Plasma homocysteine increases, often to very high levels $100 \mathrm{~mol} / \mathrm{l}$ normal upto $12 \mathrm{~mol} / \mathrm{l}$ ) with GFR $70 \mathrm{ml} /$ min. Homocysteine is metabolized by either transsulfuration with pyridoxine as cofactor or by remethylation with transcobalamin and methyltetrahydrofolate, the active form of folate, as cofactors. Deficiency of these water-soluble vitamins may develop from losses during dialysis and, coupled with poor oral intake and decreased homocysteine renal clearance, promoted hyperhomocysteinemia.

Inflammation and oxidative stress: The role of systemic inflammation and oxidative stress to the atherosclerosis development and cardiac events is currently being investigated, and a discussion is beyond the scope of this review. ESRD patients show activation of systemic inflammation and increased oxidative stress. In nondialysis dependent CKD patients, blood levels of markers of systemic inflammation and oxidative stress increase as renal dysfunction progresses. ${ }^{10}$

Immunosuppressants: Corticosteroids for immunosuppression in RTR may induce insulin resistance and hyperlipoproteinemia. Cyclosporine increases low-density lipoprotein cholesterol level in RTRs. ${ }^{11}$

\section{Is CKD itself a risk factor for CAD?}

The question of whether specific factors in CKD accelerate atherosclerosis is unanswered, but clues suggest that this may be so. CKD may accelerate atherosclerosis further in the presence of type $2 \mathrm{DM}$. In one study, diabetic RTRs had a lower ACS incidence after transplantation compared with pretransplantation. ${ }^{12}$ Vascular disease in young ESRD patients was related to inflammatory markers, hyperparathyroidism, hyperphosphatemia, and hyperhomocysteinemia but not traditional risk factors. Asymptomatic mild CKD patients in the Cardiovascular Health Study showed elevated proinflammatory and prothrombotic parameters ${ }^{10}$. These data emphasize the adverse internal environment and potential contribution of novel risk factors in the development and progression of CAD in CKD patients.

\section{Morphology of atherosclerotic plaque in CKD.} Postmortem data of CKD vessels showed increased medial thickness and smaller lumen area compared with age- and gender-matched control subjects. Control plaques were mostly fibroatheromatous, whereas CKD plaques were calcified. An electron beam computed tomography study in ESRD adults showed coronary calcification in $92 \%$; on average, calcium scores exceeded 10 -fold the 95 th percentile, the severity related to ESRD duration. ${ }^{13}$

\section{Special Issues in CKD Patients}

\section{Diagnosing ischemia and CAD symptoms}

Chest pain specificity for CAD is reduced in CKD as a result of ischemia from anemia, poorly controlled hypertension, and/or left ventricular hypertrophy, as well as, CAD. Conversely, CKD patients with $\mathrm{CAD}$ may not experience chest pain owing to diabetic or uremic neuropathy. Dyspnea on exertion is also less specific for angina as it may be secondary to anemia, volume overload, diastolic dysfunction or respiratory compensation for metabolic acidosis.

Non invasive evaluation: The electrocardiogram in CKD may display widened QRS and ST-T changes of left ventricular hypertrophy, volume overload, and electrolyte abnormalities. Peak exercise capacity may be limited by physical deconditioning, musculoskeletal problems, poorly controlled blood pressure, and anemia. Baseline electrocardiographic abnormalities and an inability to reach target heart rate in exercise electrocardiographic testing lowers sensitivity and specificity in CKD patients. Dobutamine stress-echocardiography in dialysis patients has a sensitivity of $75 \%$ to $95 \%$ and specificity of $76 \%$ to $86 \%$. Dipyridamole radionuclide stress testing has a sensitivity of $80 \%$ and specificity of $37 \%$ to $73 \% .^{14}$

\section{Invasive evaluation and contrast-induced} nephropathy (CIN)

The invasive evaluation of CAD in CKD patients is complicated by an increased CIN risk, defined as an absolute serum creatinine ( $\mathrm{sCr}$ ) increase $0.5 \mathrm{mg} /$ $\mathrm{dl}$ or relative increase $25 \%$ above baseline after contrast administration.

The most important CIN risk factors are (i) age $\geq 70$ years, (ii) pre-existing CKD, and (iii) DM. In $\mathrm{DM}$ patients with CKD, the CIN rate may exceed $25 \%$. Most studies comparing ionic high-osmolar with nonionic low-osmolar contrast in patients without high-risk features have shown no difference in renal outcome. Data suggest that non-ionic lowosmolar or isoosmolar contrast decreases 
nephrotoxicity in diabetics and CKD patients, but the effect is relatively small. One controlled randomized trial comparing normal saline hydration alone with saline and mannitol or furosemide showed that saline alone was most successful in preventing CIN. ${ }^{15}$ Several agents have been shown to be ineffective in CIN prevention, including dopamine, mannitol, furosemide, atrial natriuretic peptide, calcium channel blockers, aminophylline, and fenoldopam . Isotonic saline appears superior to hypotonic saline. Some trials in CKD patients have shown $\mathrm{N}$-acetylcysteine (an antioxidant) decreasing CIN incidence. In a meta-analysis of 805 patients, $N$-acetylcysteine reduced CIN risk by $56 \%(p=0.02)$

${ }^{16}$. Based on low cost, simple regimen, low adverse effect incidence, and clinical trial results, $N$ acetylcysteine may be used for CIN prevention. There still remains a need for a large trial to definitively demonstrate efficacy.

Contrast-induced nephropathy is usually nonoliguric. Treatment includes fastidious maintenance of fluid balance, avoidance of nephrotoxic agents, and monitoring of renal function and electrolyte status. Dialysis is necessary in only a small percentage $(0.4 \%$ to $0.8 \%$ of patients undergoing percutaneous coronary intervention PCI. ${ }^{17}$ Most patients recover by one to three weeks, but a small percent do not, or only partially. Patients with mild to moderate CKD who develop CIN after PCI show increased one-year mortality ( $45 \%$ if dialysis is required, $35 \%$ if dialysis is not required, and $19 \%$ if $\mathrm{sCr}$ does not rise, $p<0.001) .{ }^{18}$

Reliability of Biomarkers for Diagnosing AMI in CKD: Creatine kinase-myocardial band (CK-MB) elevations have been found on routine testing in dialysis patients without clinical or electrocardiographic evidence of AMI. It is unclear whether these elevations represent false positives, ongoing myocardial damage, or reduced CK-MB clearance. Troponin measurement may have similar limitations. In one study, cardiac troponin was found to be the most consistent AMI marker in all CKD strata including dialysis patients and was more sensitive and had a higher negative predictive value than myoglobin and CK-MB. However, sporadic or persistent elevations have also been seen in troponin $\mathrm{T}$ and $\mathrm{I}$ in asymptomatic patients.
Predicting prognosis with biomarkers: Studies have evaluated the relationship of biomarkers to prognosis in ACS, post-PCI, and asymptomatic CKD patients. In 7,033 ACS patients in the Global Utilization of Streptokinase and Tissue Plasminogen Activator for Occluded Arteries (GUSTO) IV trial, troponin T predicted increased AMI or death risk at one month regardless of creatinine clearance (CCr). ${ }^{19}$ The CK-MB elevation after PCI in CKD patients is associated with increased mortality and in-hospital complications.

An increase in predialysis serum troponin $\mathrm{T}$ and/or I was associated with a twofold to fivefold increase in two-year mortality in asymptomatic HD patients. In HD patients without ACS, troponin T correlated with left ventricular mass and all-cause and cardiovascular mortality. Further, in such patients, increasing troponin $\mathrm{T}$ and C-reactive protein levels were independently associated with an increased risk of death, and their combination predicted the highest death risk. The exact role of troponin testing for risk stratification of asymptomatic $\mathrm{HD}$ patients is attractive but undefined. Also unclear is the appropriate evaluation and management of CKD patients with asymptomatic serum CK-MB and/or troponin elevation.

CAD screening in renal transplant candidates: Cardiovascular events cause $35 \%$ to $50 \%$ of all deaths after renal transplantation. ${ }^{20}$ The purpose of CAD screening is two-fold:

1) To determine surgical risk, and

2) To estimate survival after transplantation.

It should be noted that there are no large-scale trials demonstrating that "prophylactic" revascularization improves long-term outcome after transplantation. Because there is a limited organ supply and patients without CAD are likely to have better long-term outcome, should screening exclude CAD patients from transplantation?

Alternatively, should aggressive screening and coronary revascularization be performed to optimize long-term outcome after transplantation for patients who survive the revascularization procedure? What is clear is that patients who manifest ischemia on pre-transplant stress testing have a higher cardiac event and mortality risk in long-term post- 
transplantation follow-up. ${ }^{21}$ These data emphasize the quandary on how to evaluate and treat these patients.

The American Society of Transplant Physicians has formulated CAD screening guidelines. Transplant candidates with angina and diabetics with evidence of ischemia should usually undergo pre-transplant coronary angiography without prior noninvasive testing. Routine pre-transplant coronary angiography in asymptomatic diabetics is uncertain. For asymptomatic patients with CAD by history or multiple risk factors, noninvasive testing may help in assessing the post-transplant cardiac risk. If the stress test is abnormal, coronary angiography is recommended. American Society of Transplant Physicians guidelines suggest revascularization before transplantation in patients with "critical" lesions based on a small randomized trial in 26 asymptomatic diabetics. ${ }^{21}$

\section{Optimizing medical management.}

There is underutilization of aspirin, beta-blockers, angiotensin-converting enzyme inhibitors (ACEI), glycoprotein IIb/IIIa receptor antagonists, diagnostic coronary angiography, thrombolytic therapy, and PCI in CKD patients with AMI or ACS. This may relate to physician concern regarding bleeding risk, worsening of renal function, lack of evidence for use of certain drugs, associated co-morbidities, and generally worsened outcomes in CKD patients. Dose adjustment in cardiac medications may be necessary.

\section{Anticoagulation}

Management of ACS in CKD patients is the same as in the general population with few exceptions. Aspirin is recommended, although no prospective efficacy or safety data have dealt specifically with CKD patients. Unfractionated heparin is preferred over low molecular-weight-heparin, which may accumulate in renal failure and for which adequate data are unavailable. Direct thrombin inhibitors are cleared partially by the kidney, urging caution until more data are available. A metaanalysis suggests that at least for bivalirudin, the drug is equally, or more, effective than unfractionated heparin and produces less bleeding. ${ }^{22}$ Safety data on glycoprotein IIb/IIIa inhibitors are limited as a result of the exclusion of CKD patients from most clinical trials. Among mild to moderate CKD patients in the
Platelet-Receptor Inhibition for Ischemic Syndrome Management in Patients Limited by Unstable Signs and Symptoms (PRISM-PLUS) trial, tirofiban plus heparin was well tolerated and effective in reducing ACS complications.

Among mild CKD patients in the Enhanced Suppression of the Platelet IIb/IIIa Receptor with Integrilin Therapy (ESPRIT) trial, bleeding risk and treatment effect with eptifibatide were similar to that in patients with normal renal function. ${ }^{23}$

\section{Management of Hypertension}

The seventh report of the Joint National Committee on prevention, detection, evaluation, and treatment of high blood pressure recommends that CKD patients should have a blood pressure $130 / 80 \mathrm{~mm}$ Hg. ${ }^{24}$ The National Kidney Foundation recommends 125/75 mm Hg for CKD patients with proteinuria 1 g/day. However, the relationship between blood pressure and mortality in HD patients may exhibit a "U"-shaped distribution wherein not only high (180 mm Hg) but also low (110 mm Hg) systolic blood pressure is associated with increased mortality. ${ }^{25}$

Hypertension in CKD patients, especially those on dialysis, is volume-dependent. Hence maintenance of fluid balance is paramount. Examination of neck veins, edema, and body weight can aid in managing fluid status.

ACEI Angiotensin-converting enzyme inhibitors decreased 30-day mortality (relative risk 0.64) in dialysis patients with AMI, an effect similar to nondialysis patients. Angiotensin-converting enzyme inhibitors decrease the progression of nephropathy in type 1 and 2 diabetes and non-diabetic renal disease. ${ }^{26}$ In the Heart Outcomes Prevention Evaluation (HOPE) trial, risk reduction for cardiovascular death, all-cause mortality, and heart failure hospitalizations with ramipril was greater for CKD than non-CKD patients. ${ }^{27}$ These agents should be used cautiously because they may induce hyperkalemia in nondialysis patients with mild to severe CKD.

Angiotensin Receptor Blockers: Renoprotection from angiotensin receptor blockers has been demonstrated in CKD patients but cardioprotection has not. Renoprotection appears to be independent of blood pressure reduction. In a randomized trial of 1,513 CKD patients with type $2 \mathrm{DM}$, nephropathy 
progression was reduced by losartan but cardiovascular death incidence was similar to placebo $^{28}$. In a trial of irbesartan, amlodipine, or placebo in 1,715 CKD patients with hypertension and type $2 \mathrm{DM}$, irbesartan afforded renoprotection but not cardioprotection. ${ }^{29}$ Based on lack of proven cardioprotective effect of angiotensin receptor blockers in CKD patients, ACEI are preferred when possible.

Beta-Blockers: Beta-blockers appear to retain their cardioprotective effects in CKD patients. In an analysis of a Medicare database of over 200,000 mild CKD patients, there was a $35 \%$ reduction in mortality with beta-blockers .

Treatment of Hyperlipidemia: Target serum low-density lipoprotein cholesterol in $\mathrm{CAD}$ patients is $100 \mathrm{mg} / \mathrm{dl}$. Statin dose reduction is required in RTRs taking cyclosporine or tacrolimus. It is not clear whether isolated hypertriglyceridemia or low levels of high-density lipoprotein cholesterol should be treated with drugs in $\mathrm{CKD}$ patients.

Treatment of Hyperhomocysteinemia: Recommended daily allowances of folate ( $5 \mathrm{mg} /$ day), transcobalamin $(0.4 \mathrm{mg} /$ day $)$, and pyridoxine $(50 \mathrm{mg} /$ day) normalize homocysteine level in mild to moderate CKD patients and RTRs, but only mildly affect homocysteine levels in dialysis patients. It seems reasonable to normalize plasma homocysteine if possible.

\section{Management of Anemia:}

Anemia may increase angina severity and left ventricular hypertrophy and decrease exercise tolerance, and its correction improves these abnormalities. The Normal Hematocrit Trial showed that patients with ESRD and CAD or heart failure treated with erythropoietin to a target hematocrit of $42 \%$ had a higher risk ratio (RR) (1.3) for the end points of death or nonfatal AMI compared with a targeted hematocrit of $30 \% .{ }^{30}$ Alternatively, a large Medicare study of HD patients using erythropoietin demonstrated decreased risk of cardiac mortality with a hematocrit of $30 \%$ to $33 \%$, and an even lower risk with $33 \%$ to $36 \%{ }^{31}$

\section{Role of Antioxidants:}

Antioxidants for cardioprotection have demonstrated conflicting results. The Secondary Prevention with Antioxidants of Cardiovascular disease in end stage renal disease (SPACE) trial demonstrated that vitamin $\mathrm{E}$ use in $\mathrm{HD}$ patients was associated with a $54 \%$ decrease in the combined end point of AMI, ischemic stroke, symptomatic peripheral vascular disease, and unstable angina. ${ }^{32}$ Other studies have not demonstrated a beneficial effect of vitamin $\mathrm{E}$. $\mathrm{N}$-acetylcysteine in HD patients decreased the composite end point of fatal/nonfatal AMI, cardiovascular death, need for revascularization, ischemic stroke, and symptomatic peripheral vascular disease versus placebo ( $28 \%$ vs. $47 \%$, p 0.03). ${ }^{33}$ Although efficacy trials with antioxidants have been disappointing in non-CKD patients, the increased oxidant stress in CKD may provide the environment for antioxidants to be cardioprotective. Clearly more data are required before any antioxidant can be recommended.

\section{Revascularizing CKD patients with PCI}

There is a striking lack of comparison of CAD treatments in CKD patients. Small studies using balloon angioplasty in HD patients have shown initial angiographic success of $56 \%$ to $96 \%$ with high re-stenosis rates ( $60 \%$ to $81 \%)$. Procedural advances and stent use have produced better angiographic success rates (90\%) and lower re-stenosis rates (31\% to $36 \%) .{ }^{34}$ Drug-eluting stents may reduce restenosis rates further, although data are currently unavailable.

Mortality risk during PCI hospitalization increases with CKD as well as DM and appears additive. The CKD patients have higher one-year mortality after PCI than non-CKD patients, a trend observed through four-year follow-up. ${ }^{35}$ Percutaneous coronary intervention use in AMI showed a higher 30-day death rate $(7.5 \%)$ in CKD versus non-CKD patients $\left(0.8 \%, \mathrm{p}\right.$ 0.0001). ${ }^{36}$ The CKD patients undergoing saphenous vein graft interventions also show a higher in-hospital and one-year mortality. ${ }^{37}$ Chronic kidney disease patients with ST-segment elevation AMI showed a lower 30-day mortality with thrombolysis (8.3\%) than PCI (37.1\%, p 0.04), ${ }^{38}$ emphasizing the uncertainty of the preferred AMI treatment in CKD patients.

\section{Coronary Artery Bypass Graft Surgery (CABG):} Coronary artery bypass graft surgery perioperative mortality in dialysis patients is approximately $7 \%$ to $10 \%$, at least three to four times non-CKD patients, and five-year mortality is estimated at $48 \%$ versus $15 \%$ in non-CKD patients. ${ }^{39}$ Most studies are retrospective, have small sample size, and are 
unadjusted. In studies with adjustment, CKD remains a highly significant predictor for decreased long-term survival. ${ }^{40}$ Not unexpectedly, HDdependent diabetics suffer worse long-term outcomes after CABG than non-diabetics. Coronary artery bypass graft surgery outcomes in mild or moderate CKD patients are limited. Chronic kidney disease patients (vs. non-CKD patients) had longer inhospital and intensive care unit stay and more frequent postoperative dialysis. ${ }^{41}$ In a prospective study of 2,222 mild CKD patients, $7.7 \%$ had postoperative renal dysfunction associated with prolonged intensive care unit and hospital stays and increased mortality. ${ }^{42}$ In another analysis of mild to moderate CKD patients, inhospital CABG mortality was $11 \%$ and actuarial survival at 10 years was $32 \%$, similar to dialysis patients. ${ }^{43}$

\section{Comparison of CABG and PCI:}

Studies comparing CABG with PCI in HD patients are all non-randomized and retrospective. There may be an increased perioperative mortality but better long-term survival and freedom from angina with CABG compared with balloon angioplasty. A preliminary report from a large prospective trial comparing stenting and CABG in patients with multi-vessel disease suggests similar outcomes. A non-randomized study in CKD patients with estimated GFR $60 \mathrm{ml} / \mathrm{min}$ with ACS showed that PCI was associated with improved survival compared with CABG or medical therapy .

\section{Conclusions:}

Patients with ESRD have more than CAD risk equivalent status in their baseline CAD risk assessment.This review has highlighted that $\mathrm{CAD}$ is widely prevalent in $\mathrm{CKD}$ and that $\mathrm{CAD}$ patients with CKD have a worsened prognosis. The current challenge is to study the CAD patient with CKD in prospective randomized trials to provide an evidencebased approach to therapy. In the absence of such information, aggressive control of $\mathrm{CAD}$ risk factors and timely intervention for symptomatic CAD is suggested.

\section{References:}

1. Sarnak MJ, Levey AS, Schoolwerth AC, et al. Kidney disease as a risk factor for development of cardiovascular disease. Circulation 2003; 108: 2154-69.

2. Foley RN, Parfrey PS, Sarnak MJ. Clinical epidemiology of cardiovasculardisease in chronic renal disease. Am J Kidney Dis 1998; 32Suppl 3: 112-9.
3. Wright RS, Reeder GS, Herzog CA, et al. Acute myocardial infarction and renal dysfunction: a highrisk combination. Ann Intern Med 2002; 137: 563-70.

4. Al Suwaidi J, Reddan DN, Williams K, et al. Prognostic implications of abnormalities in renal function in patients with acute coronary syndromes. Circulation 2002; 106: 974-80.

5. Shlipak MG, Heidenreich PA, Noguchi H, Chertow GM, Browner WS, McClellan MB. Association of renal insufficiency with treatment and outcomes after myocardial infarction in elderly patients. Ann Intern Med 2002; 137: 555-62.

6. U.S. Renal Data System. 2001 Annual Data Report. Bethesda, MD: 2001.

7. Wu M-S, Yu C-C, Yang C-W, Wu C-H, Huang J-Y, Hong J-J, Chiang C-Y, Huang C-C, Leu M-L: Poor predialysis glycaemic control is a predictor of mortality in type II diabetic patients on maintenance haemodialysis. Nephrol Dial Transplant 1997; 12: 2105-10.

8. Ganesh SK, Stack AG, Levin NW, Hulbert-Shearon TE, Port FK. Association of elevated serum phosphate, calcium x phosphate product, and parathyroid hormone with cardiac mortality risk in chronic hemodialysis patients. J Am Soc Nephrol 2001; 12: 2131- 8.

9. Moustapha A, Naso A, Nahlawi M, et al. Prospective study of hyperhomocysteinemia as an adverse cardiovascular risk factor in end-stage renal disease. Circulation 1998; 97: 138-41.

10. Shlipak MG, Fried LF, Crump C, et al. Elevations of inflammatory and procoagulant biomarkers in elderly persons with renal insufficiency. Circulation 2003; 107: 87-92.

11. Taylor DO, Barr ML, Radovancevic B, et al. A randomized, multicenter comparison of tacrolimus and cyclosporine immunosuppressive regimens in cardiac transplantation: decreased hyperlipidemia and hypertension with tacrolimus. J Heart Lung Transpl 1999; 18: 336-45.

12. Hypolite IO, Bucci J, Hshieh P, et al. Acute coronary syndromes after renal transplantation in patients with end-stage renal disease resulting from diabetes. Am $J$ Transplant 2002; 2: 274-81.

13. Schwarz U, Buzello M, Ritz E, et al. Morphology of coronary atherosclerotic lesions in patients with end-stage renal failure. Nephrol Dial Transpl 2000; 15: 218-23.

14. Reis G, Marcovitz PA, Leichtman AB, et al. Usefulness of dobutamine stress echocardiography in detecting coronary artery disease in end-stage renal disease. Am J Cardiol 1995; 75: 707-10.

15. Solomon R, Werner C, Mann D, D'Elia J, Silva P. Effects of saline, mannitol, and furosemide to prevent acute decreases in renal function induced by radiocontrast agents. N Engl J Med 1994; 331: 1416-20.

16. Birck R, Krzossok S, Markowetz F, Schnulle P, van der Woude FJ, Braun C. Acetylcysteine for prevention of 
contrast nephropathy: meta-analysis. Lancet 2003; 362: 598-603.

17. Freeman RV, O'Donnell M, Share D, et al. Nephropathy requiring dialysis after percutaneous coronary intervention and the critical role of an adjusted contrast dose. Am J Cardiol 2002; 90: 1068-73.

18. Gruberg L, Mintz GS, Mehran R, et al. The prognostic implications of further renal function deterioration within 48 hours of interventional coronary procedures in patients with pre-existent chronic renal insufficiency. J Am Coll Cardiol 2000; 36: 1542-8.

19. Aviles RJ, Askari AT, Lindahl B, et al. Troponin T levels in patients with acute coronary syndromes, with or without renal dysfunction. N Engl J Med 2002; 346: 2047-52.

20. Dimeny EM. Cardiovascular disease after renal transplantation. Kidney Int 2002; 61: s78-84.

21. Patel AD, Abo-Auda WS, Davis JM, et al. Prognostic value of myocardial perfusion imaging in predicting outcome after renal transplantation. Am J Cardiol 2003; 92: $146-51$.

22. Chew DP, Bhatt DL, Kimball W, et al. Bivalirudin provides increasing benefit with decreasing renal function: a meta-analysis of randomized trials. Am J Cardiol 2003; 92: 919-23.

23. Reddan DN, O'Shea JC, Sarembock IJ, et al. Treatment effects of eptifibatide in planned coronary stent implantation in patients with chronic kidney disease (ESPRIT Trial). Am J Cardiol 2003; 91: 17-21.

24. Chobanian AV, Bakris GL, Black HR, et al. The seventh report of the Joint National Committee on prevention, detection, evaluation, and treatment of high blood pressure. JAMA 2003; 289: 2560-72.

25. Zager PG, Nikolic J, Brown RH, et al. "U" curve association of blood pressure and mortality in hemodialysis patients. Kidney Int 1998; 54: 561-9.

26. Lewis EL, Hunsicker LG, Bain RP, Rohde RD. The effect of angiotensin-converting enzyme inhibition on diabetic nephropathy. N Engl J Med 1993; 329: 1456-62.

27. Mann JFE, Gerstein JC, Pogue J, et al. Renal insufficiency as a predictor of cardiovascular outcomes and the impact of ramipril: the HOPE randomized trial. Ann Intern Med 2001; 134: 629-36.

28. Brenner BM, Cooper ME, de Zeeuw D, et al. Effects of losartan on renal and cardiovascular outcomes in patients with type 2 diabetes and nephropathy. $N$ Engl J Med 2001; 345: 861-9.

29. Lewis EJ, Hunsicker LG, Clarke WR, et al. Renoprotective effect of the angiotensin receptor antagonist irbesartan in patients with nephropathy due to type 2 diabetes. $N$ Engl J Med 2001; 345: 851-60.

30. Besarab A, Bolton WK, Browne JK, et al. The effects of normal as compared with low hematocrit values in patients with cardiac disease who are receiving hemodialysis and epoietin. N Engl J Med 1998; 339: 584-90.

31. Ma JZ, Ebben J, Xia H, Collins AJ. Hematocrit level and associated mortality in hemodialysis patients. J Am Soc Nephrol 1999; 10: 610-9.

32. Boaz M, Smetana S, Weinstein T, et al. Secondary prevention with antioxidants of cardiovascular disease in end stage renal disease (SPACE): randomized placebo-controlled trial. Lancet 2000; 356: 1213-8.

33. Tepel M, van der Giet M, Statz M, Jankowski J, Zidek W. The antioxidant acetylcysteine reduces cardiovascular events in patients with end-stage renal disease: a randomized controlled trial. Circulation 2003; 107: $992-5$.

34. Le Feuvre C, Dambrin G, Helft G, et al. Clinical outcome following coronary angioplasty in dialysis patients: a case control study in the era of coronary stenting. Heart 2001; 85: 556-60.

35. Feuvre CL, Borentain M, Beygui F, Helft G, Batisse JP, Metzger JP. Comparison of short and long-term outcomes of coronary angioplasty in patients with and without diabetes mellitus and with and without hemodialysis. Am J Cardiol 2003; 92: 721-5.

36. Best PJM, Lennon R, Ting HH, et al. The impact of renal insufficiency on clinical outcomes in patients undergoing percutaneous coronary interventions. J Am Coll Cardiol 2002; 39: 1113-9.

37. Gruberg L, Weissman NJ, Pichard AD, et al. Impact of renal function on morbidity and mortality after percutaneous aortocoronary saphenous vein graft intervention. Am Heart $J$ 2003; 145: 529-34.

38. Dragu R, Behar S, Boyko V, Kapeliovich M, Rispler S, Hammerman H. Should primary percutaneous coronary intervention be the preferred method of myocardial perfusion for ST elevation acute coronary syndromes in patients with renal failure? Circulation 2003; 108: IV-614.

39. Batiuk TD, Kurtz SB, Oh JK, Orszulak TA. Coronary artery bypass operation in dialysis patients. Mayo Clin Proc 1991; 66: 45-53.

40. Dacey LJ, Liu JY, Braxton JH, et al. Long-term survival of dialysis patients after coronary bypass grafting. Ann Thorac Surg 2002; 74: 458-63.

41. Rao V, Weisel RD, Buth KJ, et al. Coronary artery bypass grafting in patients with non-dialysis-dependent renal insufficiency. Circulation 1997; 96 (Suppl 2): 38-45.

42. Mangano CM, Diamondstone LS, Ramsay JG, Aggarwal A, Herskowitz A, Mangano DT. Renal dysfunction after myocardial revascularization: risk factors, adverse outcomes, and hospital resource utilization. Ann Intern Med 1998; 128: 194-203.

43. Nakayama Y, Sakata R, Ura M, Itoh T. Long-term results of coronary artery bypass grafting in patients with renal insufficiency. Ann Thorac Surg 2003; 75: 496-500. 